1895. His success in this work is well attested by the following extract from the report of his successor:

"It was apparent that to bring about the required changes would entail an immense amount of work, and Dr. Bodington felt constrained, through the advance of years, to relinquish the labour to younger hands, and seek well-earned rest and retirement in the land of his birth, surrounded by the members of his family and his friends. In this connection I can truthfully testify that no greater general sorrow has been witnessed throughout the institution than that occasioned by the departure of the one who had taken the helm at a very trying time, and had safely piloted the hospital through some difficult passages, giving to it his best energy and utmost attention, though never in robust health, and having already arrived at that period of life when men hope to be able to forget labour and worry. Dr. Bodington's resignation was accepted by the Government, who voted him a retiring allowance for his faithful services, which terminated on February 28th, after six years of most arduous toil, during the greater part of which he was alone in the charge of the institution."

He came to England last year to visit his relations and to renew acquaintance with old friends, and he eventually settled in Paris. His death occurred after a very short illness, but his state of health had not been satisfactory for some time past.

Dr. Bodington was a man of magnificent physique and fine presence, a delightful companion, and an enthusiastic man of letters. He was for many years a member of this Association; he resigned on going to British Columbia, and was re-elected in 1895.-From the British Medical Fournal.

\title{
NOTICES BY THE REGISTRAR
}

Examination for the Nursing Certificate.

Five hundred and forty-three candidates applied for admission to the May examination for this certificate. Of this number 179 failed to satisfy the examiners, twelve withdrew, and the following were successful :

\section{ENGLAND.}

Three Counties, Hitchin.-Females : Edith Folkes, Beatrice Florence Rainbow, Lucy Devereux, Minnie Winter, Maud Rainbow. Males: Charles Michel, Isaac Bottomley.

Bucks County.-Females : Violetta Peck, Emily Hutchins, Emily Hughes, Agnes Louisa Smith, Ada May Johnson, Agnes Louisa Walton, Mary Kinsella. Male : Christopher Hicks.

Cumberland and Westmorland.-Female : Sarah Heron. Males : John Stewart, Richard Lambert.

Devon County.-Females : Ada Trenerry, Annie Elizabeth Harris, Bessie Marley, Mary Ellen Ley. Males : William Henry Ponsford, John Tuplin, Edwin Leach.

Kent County, Maidstone.-Females: Eugenie Emily Bishop, Ellen Lavinia

Morris. Male : Charles Thomas Worsel.

Kent County, Chartham.-Male: Harry Percival Hastings

Lancaster County, Rainhill.-Females : Bessie Case, Nellie Trusler, Edith Maud Alford, Alice Mansefield Caley, Lilian Riley, Annie Moss, Edith J. Poulton, Ada Foster, Sarah Smith, May Hughes, Jessie Hollingworth, Hannah Drabble, Beatrix Ethel Hudson, Emma Smith, Rachel Crilly, Alice Mary Lamb, Mary

Mason, Catherine Lamb. Males : James Renshaw, William Leonard Gill, James Everitt, William Stones, Archibald Osman Wynne, Harry Harding, William Charles Boddy, Leonard Roberts Kingdom, Walter Hague.

London County, Bexley.-Females : Annie Berry, Alice Musto. Males : Arthur Watson, Edwin Robert Blackman, Ernest Gordon Clark, Alfred Williams, Thomas Foster.

London County, Cane Hill.-Females: Lily Annie Ashby, Esther New, Eliza-

beth Rees. Males : John McKeown, George Griffith.

London County, Claybury. - Females: Amy Louisa Lelliott, Catherine Helen 
Henson, Alma Augusta Annie Holtzhansen, Alice Mary Ayres, Bertha Skinner, Ethel Ellis, Clara Charlotte Webb, Beatrice Ida Shirley. Male : Walter Harry Dew.

Middlesex County.-Females: Annie Berry, Ida Caroline Reakes, Annie Maxwell, Alice Rebecca Mawson, Anastasia Maria McDonald, Harriet Almma Spurling. Males : William Henry Russell, George Sharpe, George Bowmer, Arthur Thomas Boxall.

Warneford Asylum, Oxford.-Females: Kate Alice Wilkins, Agnes Thomson.

Surrey County, Brookwood.-Females: Harriet Annie Baker, Frances Brodribb,

Edith Frances Crouch, Emeline Ada Driver, Amy Elizabeth Platt, Maud Rosaline

Griffin, Margarat Walters. Males : Henry Lowe, Charles Arthur Woodcock,

Thomas Betteridge.

Warwick County, Hatton.-Females: Frances Rudge, Elizabeth Hinsley, Nancy

Woosnam. Males: Harry Frederick Latham, George Matthews.

Isle of Wight.-Males : William Edwards, William Henry Sneath.

North Riding, Yorks. - Female : Catherine Emeline Jackson. Males: John

Robert Peckitt, Thomas Dunn Bertram.

Menston, Yorks. - Females : Isabel Patchett, Annie Ford, Mary Greenall

Molyneux. Males : Joseph Craven Thornton, James Holmes.

South Riding, Wadsley.-Female : Mary Jane Batty. Males : John Richard

Reynolds, Charles Robert Lincoln.

City of Birmingham, Winson Green.-Female : Alice Lees. Males: William

Edward Barry, Arthur Walton.

City of Birmingham, Rubery Hill.-Females: Florence Gwendoline Wright,

Ada Collins, Harriet Ada Stringer, Annie Maria Ellis, Ellen Roberts, Harriet

Cotton, Florence Clews. Male : Arthur Thomas Horton.

Bristol City.-Female : Edith Mary Yeoman.

Hull City.-Females : Mary Emma Clark, Ada Learoyd, Kate Harrison,

Annie Louisa Credland, Kathleen Winifred McCardle. Male: George Herbert.

Newcastle City.-Females : Margaret Wilson Smith, Deborah Elizabeth

Haynes, Marian Aldis, Isabella Bulman, Mary Jane Law. Male: Walter Phillips.

Nottingham City.-Females: Mary Ellen Rawson. Males: John Henry

Stapleton, William Loach, Frank Law, Mark Tomlinson, George William Frow.

Plymouth Borough.-Females : Evelyn Hodge, Florence Ada Harper.

Portsmouth Borough.-None.

Sunderland Borough.-Females: Louise Slaney Page, Elizabeth Ann Smith.

Males: John Charles Young, William Lewis Willcock, William Allen, David Hume.

West Ham Borough.-Females: Charity Filby, Louisa Mary Maud Griffin, Jane

Waite Robinson. Males : Walter Wilkinson Hollis, James Hunt, Joseph Jennings,

William Charles Peat, George Edwin Sparkes, Samuel Frederick Robinson.

Darenth.-Female: Edith Jane Henderston.

Leavesden.-Males : Robert Watson, Stephen John Constable.

Friend's Retreat, York.-Females: Jessie Maria Pearson, Margaret Gibbon,

Christian William Gordon, Sara Christine Hearder, Edith Emily Parker, Lucy Foster Swain, Dora Cole, Eleanor Alice Wood, Annie Eliza Naylor, Hannah Mary Iveson.

Camberwell House-Females : Elizabeth E. Brazebridge, Florence Jessie Cheal, Alice C. Graham, Minnie A. Vinicombe. Males : John Henry Moore, John Lewis, Sam Hedgland Blake.

Holloway Sanatorium.-Females: Millicent Hannah Strong, Emily Lonsdale McMaster.

Redlands.-Male : Adolphus William Pulman.

St. Luke's Hospital.-Females: Violetta Bidgood, Elizabeth Potter. Males: John Barnard, William Heslop.

Haydock Lodge.-Male: William Waterhouse Cromack.

\section{WALES.}

Abergavenny County.-Females : Mary Hannah Davies, Nellie Oakley, Edith Price, Eleanor Williams, Margaret Ann Williams. Males: Michael Doolan, 
William Henry Jones, John Preece, Alfred Price, George Richard William Rosser Edward Williams.

Glamorgan County.-Females: Elizabeth James, Clara May Jones, Edith Maud Poyntz, Martha Ann Rowlands, Mary Ann Waldin, Hannah Elizabeth Wherritt, Elizabeth Tarr. Males : Rees John Davies, John Edmunds, William Hopkins, John Bevan Preece, David Price, William R. Rogers, George Perry, John Thomas.

\section{SCOTLAND.}

Argyle and Bute District.-Female: Nellie Sherrie.

Aberdeen Royal Asylum.-Females: Elsie Carr Wilson, Helen Wilson, Bella

Valentine McDonald, Georgina Black. Males : Peter Duncan, George Skene.

Dundee Royal.-Females: Margaret Crocket McKenzie, Jane Hay.

Edinburgh Royal Asylum.-Females: Annie McLarty, Maggie Campbell, Isabella Nicol, Janet Smith, Margaret McDonald, Jane Malcolm Ashton.

Gartnavel Royal.-Females : Ellen Mary Hawkins, Elizabeth Mearns, Isa Craig

Brown, Mary Bissett. Males: William Ingram, William Duncan.

Gartloch Asylum.-Females : Marie Walker, Grace Thomson, Annie McBride,

Elizabeth Eleanor McPhillips, Martha Henry, Anne Clare Brennan. Males: James Gibson, John Findlay.

Inverness District Asylum.-Females : Ella Munro, Katherine Shaw Mackay

Cecilia Elizabeth Curran. Male: Alexander Macquarrie.

Lanark District. - Females: Fanny Dawson, Marjory Lindsay, Elizabeth $\mathbf{H}$.

Lowe, Mary Bleakley, Agnes S. Murray, Mabel N. Matheson, Lizzie Tait, Mary

S. Muir. Males : Thomas Johnston, Hector MacPhail, Andrew Adam.

James Murray's Royal.-Females : Margaret Sutherland, Elizabeth Corbett. Male : James Forbes Stalker.

Perth District Asylum.-Females: Thomasina Cramb, Annie Thomson.

Roxburgh District Asylum.-Female : Elizabeth Anne Telfer. Males : Alexander Cameron Grant, James Marr.

Smithston Asylum.-Female: Sara Morrison. Males: James M'Kaig, John

Frew, David Skelton.

Stirling District Asylum.-Female: Mary Elizabeth Smith.

Woodilee Lensie Asylum.-Females: Isa Allen Stewart, Anna Elizabeth Hay,

Elizabeth Stevenson MacIntyre, Bessie Dick Marshall, Mary Barnwell Young, Jane

Gemmell Brennan. Male : George Watt.

\section{IRELAND.}

Armagh District Asylum.-Females: Bridget McMahon, Sarah Mullan, Adelaide Hewitt, Ellen Hughes. Males : John McMahon, John Molloy, Robert Baird, James Devlin, John O'Farrell.

Cork District.-Females : Kate Coghlan, Hester Murphy, Mary Rearden, Kate Walsh, Maud Leyne, Katherine M. Reynolds. Males : John O'Callaghan, Cornelius Reardon.

Clonmel District.-Male: William O'Gorman.

Down District.-Females : Mary Harkin, Elizabeth Wilson. Males: William John McComb, William Conway.

Limerick District.-Female : Helena McEvoy. Males: Joseph Slattery, Richard Galligan.

Londonderry District.-Female: Matilda McConnell. Males : James Kyle, Hugh Henry, Hugh Margay.

Richmond District Asylum.-Females: Ellen Kelly, Teresa Pollock, Kate Kinsella, Bridget Rowan, Bridget Connell, Julia Howell, Annie Elizabeth Doran, Elizabeth R. McGee, Lizzie Johnston, Susette Edith Herd, Bridget Boland, Lizzie Mary Higgins, Emily Alice Fogartey, Margaret Owens, Margaret Lynch, Mary Jane Qooney. Males : James Napier, Peter Donnelly, Peter O'Loughlin, Thomas Barry, Stephen Maher.

Waterford District.-Females : Kate Quinlan, Mary Burke.

St. Patrick's Hospital, Dublin.-Female: Agnes Bailey. Males : John Joseph Piggott, Patrick John Fagan, John O'Neill. 
St. Edmunsbury.-Females : Dorothy Kent, Mary Bridget Pender. Dr. Doroson's, Maryoille. - Females : Jennie Hewitt, Margaret Vaughan, Margaret Cullen.

\section{ENGLAND.}

Somerset and Bath.-Females: Louisa Ivy Hancock, Annie Bickell. Male: William Charles Hawkins.

City of London.-Males : Edward James Thorp, Walter John Figg.

West Sussex.-Males: James Hayward Strudwick, James F. Cragg, Arthur Turner.

London County, Claybury.-Female: Jennie Anderson King.

The following is a list of the questions which appeared on the paper: 1 . What do you understand by fresh air? What is its composition? What impurities are found in air which has been respired ? 2. What is the pelvis? Name the bones forming it. What organs does it contain? What are their relative positions? 3. What should be noted about the urine of patients? What are the different conditions which may lead to incompetence of the bladder $P$ 4. In what cases may suicidal attempts be made? What are the most frequent modes in which patients attempt suicide, and how do you guard against it $P$ 5. To what special risks of injury are epileptic patients liable? How would you guard against these risks ? 6. How are bedsores caused ? In what cases are they liable to occur? How would you prevent their occurrence? 7. How would you treat an attack of $(a)$ apoplexy; (b) syncope (fainting); (c) choking? 8. What are the special points to be attended to in feeding paralytic and helpless patients? 9. State briefly how you would guard against and discover escapes; what precautions would you take aganist homicidal impulses; and what do you understand by special observation. 10. Into how many classes may poisons be roughly divided $P$ Name them, give examples of each, and state what steps you would take in cases of suspected poisoning directly the doctor had been called, but before his arrival.

\section{Examination for Nursing Certificate.}

The next examination will be held on Monday, November 3 rd, 1902.

Note.-As the names of some of the persons to whom the Nursing Certificate has been granted have been removed from the register, employers are requested to refer to the Registrar in order to ascertain if a particular name is still on the roll of the Association. In all inquiries the number of the certificate should be given.

Examination for the Professional. Certificate.

The next examination for the Certificate in Psychological Medicine will be held on Thursday, July izth, 1902.

\section{Gaskell. Prize.}

The next examination for the Gaskell Prize will be held on Friday, July 18th, 1902.

\section{NOTICES OF MEETINGS.}

\section{Medico-Psychological Association.}

The Sixty-first Annual Meeting of the Association will be held at the Medical Institution, Hope Street (corner of Mount Pleasant), Liverpool, on Thursday and Friday, July 24th and 25th, 1902, under the Presidency of Dr. J. Wiglesworth. There will be a meeting of Committees as follows on Thursday, July 24th, before the Annual Meeting : Educational Committee 9 a.m., Rules Committee 9.30 a.m. Parliamentary Committee 9.45 a.m., Council Meeting 10 a.m.

The Annual Meeting commences at II a.m. on Thursday, when the usual business of the Association will be transacted. 